\title{
Polymorphism of the methylenetetrahydrofolate reductase gene association with homocysteine and ischemic stroke in type 2 diabetes
}

\author{
Jia-Zhong Sun, Yancheng Xu, Hongyun Lu', Yilian Zhu \\ Departments of Endocrinology, Zhongnan Hospital, Wuban University, Wuban - 430 071, ${ }^{1}$ The Fifth Affliated Hospital of \\ SunYat-sen University, Zhubai, 519 000, Guangdong, China
}

\begin{abstract}
Background: Ischemic stroke is a freeuent heterogeneous multifactorial disease. A number of genetic mutations and environmental factors have been implicated. A polymorphism in the gene for methylenetetrahydrofolate reductase (MTHFR) has been reported to be associated with hyperhomocysteinemia a risk for atherosclerotic vascular diseases. Aim: A cross-sectional study was performed to determine the relationship between the gene polymorphism for MTHFR and ischemic stroke in type 2 diabetes mellitus. Materials and Methods: Of the 215 unrelated patients with type 2 diabetes mellitus recruited, 119 patients had ischemic stroke, Control group included 142 healthy subjects. The genotype of the subjects for the C677T polymorphism of MTHFR was analyzed by using polymerase chain reactionrestriction fragment length polymorphism (PCR-RFLP) followed by Hinfl digestion. Plasma total homocysteine (Hcy) levels were measured using high-performance lieuid chromatography (HPLC) with fluorescence detection. Results: The genotype distribution did not differ between the control subjects and type 2 diabetic patients $(P>0.05)$. Plasma homocysteine levels were markedly higher in diabetic patients with TT genotype than those with CC or CT genotype $(P>0.05)$. Ischemic stroke was more frequently observed in type 2 diabetic patients with the TT genotype than in those with the $\mathrm{CT}$ and $\mathrm{CC}$ genotype (odds ratio $=4.04,95 \% \mathrm{Cl}=1.95-8.34$, $P=0.0036)$. Logistic regression analysis revealed that the C677T mutation of MTHFR gene was independently associated with ischemic stroke in type 2 diabetes. Conclusion: MTHFR C677T gene polymorphism associated with a predisposition to hyperhomocysteinemia could constitute a useful predictive marker for ischemic stroke in type 2 diabetic Chinese patients.
\end{abstract}

Address for correspondence:

Prof. Jia-Zhong Sun,

Department of Endocrinology,

Zhongnan Hospital, Wuhan

University, Wuhan - 430071 , China.

E-mail: sjz300@163.com

PMID: 19934557

DOI: $10.4103 / 0028-3886.57808$
Key words: Type 2 diabetes mellitus, ischemic stroke, methylenetetrahydrofolate reductase, polymorphism

\section{Introduction}

Ischemic stroke in patients with type 2 diabetes mellitus is a major cause of morbidity and mortality. However, the mechanisms for the predisposition are still unclear. The high risk for ischemic stroke is partly explained by hyperglycemia, arterial hypertension, and dyslipidaemia. ${ }^{[1,2]}$ All of these risk factors have strong genetic components, ${ }^{[3]}$ which may contribute to macrovascular as well as microvascular complications in diabetes mellitus. Genetic determinants may accelerate diabetes-induced atherosclerosis. Identification and characterization of gene variants that play such a role may allow improved prognostication, therapy, and prevention. 
There is a large body of observational, biologically plausible evidence linking hyperhomocysteinemia in a dosedependent fashion to atherosclerotic vascular morbidity and mortality, including stroke ${ }^{[3,4]}$ Hyperhomocysteinemia appears to be a modifiable risk factor for ischemic stroke. Methylenetetrahydrofolate reductase (MTHFR) plays an important role in homocysteine (Hcy) metabolism. MTHFR provides the methyl group for methionine synthase, methylating homocysteine to methionine. Plasma levels of Hcy are governed by both genetic and nutritional factors such as vitamins $\mathrm{B}_{12^{\prime}} \mathrm{B}_{6^{\prime}}$, and folic acid. A common missense mutation in the MTHFR gene, a C-to-T substitution at nucleotide 677 , is responsible for reduced MTHFR activity and is associated with moderate increase in plasma Hcy concentrations. C677T homozygous genes have significantly elevated plasma Hcy levels. ${ }^{[4]}$ It is known that the frequency of the homozygous mutant is different in different ethnic groups.$^{[5]}$ The purpose of our study was to investigate the distribution of C677T MTHFR in type 2 diabetic patients and the influence of mutant genotype on ischemic stroke.

\section{Materials and Methods}

\section{Subjects}

After the approval of the ethics committee and informed consent, 215 unrelated, Chinese, type 2 diabetic patients were recruited from Wuhan University Hospital, Wuhan, China. Diabetes mellitus was diagnosed and classified according to the criteria of the World Health Organization. Of the 215 diabetic patients, 119 patients had ischemic stroke. The diagnosis of ischemic stroke was considered when neurological deficits were accompanied by the corresponding abnormalities on computed tomography (CT) of the brain. Neurological and CT findings were interpreted by at least two independent experienced neurologists. Patients with cerebral hemorrhage and atrial fibrillation were excluded. Similarly subjects with overt nephropathy with serum creatinine above $178 \mu \mathrm{mol} / \mathrm{L}$, were also excluded. Classification of stroke was based on the criteria proposed by the National Institute of Neurological Disorders and Stroke Ad Hoc Committee. ${ }^{[6]}$ The remaining 96 subjects without stroke were grouped under diabetes without ischemic stroke. Control group $(n=142$, mean age: $52.3 \pm 6.1$, sex ratio $M / F=82 / 60$ ) included healthy non-diabetic subjects and were recruited from the industry health clinics in Wuhan. The controls were unrelated to diabetic patients and had normal resting electrocardiogram and no symptoms suggestive of coronary artery disease. Normoglycemia was defined as having a fasting glucose level less than $6.1 \mathrm{mmol} / \mathrm{L}$ on two consecutive occasions.

\section{Clinical analysis}

The duration of diabetes mellitus was determined by reviewing the clinical history and the medical records. Arterial blood pressure was measured from the right arm in the supine position after 10 minutes of rest in lying position. Hypertension was defined as a systolic blood pressure $\geq 140 \mathrm{mmHg}$, a diastolic blood pressure $\geq 90 \mathrm{mmHg}$, or the prescription of antihypertensive medication. The subjects were divided into smoking and non-smoking groups according to their current smoking status. Body mass index (BMI) was calculated by dividing the subject's weight in kilograms by the square of their height in meters.

\section{Biochemical analysis}

Venous blood was collected into EDTA-tubes after an overnight fast, using standardized protocol and equipment. Plasma was immediately prepared at $4^{\circ} \mathrm{C}$ and snap frozen and kept at $-30^{\circ} \mathrm{C}$. Total plasma homocysteine levels was measured by high performance liquid chromatography (HP1100 type) coupled with fluorescence detection (HP1046 A type). Analytical column is ODS C185 $\mu \mathrm{m}$ reversal-phase analytical column. The excitation and emission wave length is set at $385 \mathrm{~nm}$ and $515 \mathrm{~nm}$, respectively. We used a $0.1 \mathrm{mmol} / \mathrm{L}$ potassium dihydrogenphosphate buffer ( $\mathrm{pH}$ 2.1, adjusted with ortophosphoric acid) containing $10 \%$ acetonitrile as mobile phase with a flow-rate of $0.2 \mathrm{~mol} / \mathrm{min}$. The intrassay and interassay coefficients of variation for total Hcy were $3.52 \%$ and $3.63 \%$ (Sigma company Hcy standard samples as control), respectively.

Serum creatinine, cholesterol, triglycerides, high density lipoprotein-cholesterol, as well as other basic biochemical blood tests, were measured by standard chemical and enzymatic commercial methods in the hospital laboratory. Glycated hemoglobin (HbA1c) was measured by HP1100 type. Urinary albumin excretion (UAE) levels were obtained on a single sample obtained in the morning and were measured by nephelometry.

\section{Genetic analysis}

Genomic DNA was extracted from peripheral blood leukocytes by the phenol-chloroform method. Polymerase chain reaction (PCR) was performed on the genomic DNA samples with a Gene Amp PCR kit (Promega) and primers as previously reported ${ }^{[4]}$ The forward primers were 5'-TGAAGGAGAAGGTGTCTGCGGGA-3' and the reverseprimerwas5'-AGGACGGTGCGGTGAGAGTG-3'. 35 cycles $\left(94^{\circ} \mathrm{C}\right.$ for 45 seconds, $60^{\circ} \mathrm{C}$ for 45 seconds, and $72^{\circ} \mathrm{C}$ for 50 seconds) were used to amplify 198-bp products.

The 198-bp PCR product (10 ul) was digested with the restriction enzyme HinfI at $37^{\circ} \mathrm{C}$ for $3-4$ hours in the buffer recommended by the manufacturer. HinfI can recognize the C-to-T substitution in the fragments. This one nucleotide substitute corresponds to a conversion of Ala-to-Val residue in the MTHFR encoding region. The two different alleles were designated $\mathrm{T}$ (Val) and 
C (Ala), The 198-bp fragment derived from the C allele is not digested by Hinfl, whereas the fragments of the same length from the $\mathrm{T}$ allele is digested by HinfI into 175 and 23-bp fragments. Subjects homozygous for the mutation showed two DNA fragments of 175-bp and 23-bp, whereas homozygous subjects without it showed a DNA fragment of 198-bp. Heterozygous subjects showed three DNA fragments of 198-bp, 175-bp and 23-bp. The HinfI-treated PCR fragments were analyzed by $8 \%$ polyacrylamide gel electrophoresis at $85 \mathrm{w}$ for 6-8 hours and visualized with silver nitrate.

\section{Statistical analysis}

All statistical analyses were done using the SPSS statistical package, version 10.0. Distributions of continuous variables in groups were expressed as mean \pm SD and compared by Student's $t$ tests or one-way analysis of variance (ANOVA). The Hardy-Weinberg equilibrium or odds ratio (OR) and 95\% confidence interval (CI) for the presence of ischemic stroke within the MTHFR genotypes were analyzed by using the $\chi^{2}$ test. Logistic regression analysis was performed to adjust the contribution of each risk factor for ischemic stroke. Results were considered significant or nonsignificant when $P>$ or $<0.05$, respectively.

\section{Results}

In the screening of 142 healthy Chinese people, the allele frequency of the $T$ mutation was $32.0 \%$, The distribution of the three genotypes was: CC genotype, $54.9 \%$; CT genotype, $26.8 \%$; and TT genotype, $18.3 \%$. This genotype distribution was similar $\left(\chi^{2}=3.12, P>0.05\right)$ to the distribution in type 2 diabetic patients; CC genotype $43.7 \%$, CT genotype $34.0 \%$, and TT genotype $22.3 \%$ [Table 1].

When the diabetic patients were classified according to their MTHFR genotype, no statistically significant differences in clinical or metabolic variables were found among the C677T genotypes [Table 2]. The groups were also similar with regard to known risk factors for atherosclerosis, such as smoking, hypertension, hyperlipidemia, the prevalence of diabetic retinopathy, levels of urinary albumin excretion (UAE), and prevalence of overt proteinuria were similar in all groups.

The plasma Hcy levels were higher in patients with ischemic stroke when compared with patients without ischemic stroke $(22.66 \pm 3.12 v s .13 .21 \pm 3.07 \mu \mathrm{mol} / \mathrm{L}$, $P<0.001)$. There was a positive relationship between plasma Hcy level and ischemic stroke.

As showed in the Figure 1, the T allele was significantly

\begin{tabular}{|c|c|c|}
\hline \multicolumn{3}{|c|}{$\begin{array}{l}\text { Table 1: Distribution of methylenetetrahydrofolate reductase } \\
\text { genotypes in healthy people and patients with type } 2 \text { DM }\end{array}$} \\
\hline $\begin{array}{l}\text { MTHFR } \\
\text { genotype }\end{array}$ & $\begin{array}{l}\text { Normal subjects } \\
(\mathrm{n}=142) \mathrm{N}(\%)\end{array}$ & $\begin{array}{l}\text { Diabetic patients } \\
(\mathrm{n}=215) \mathrm{N}(\%)\end{array}$ \\
\hline $\mathrm{CC}$ & $78(54.9)$ & $94(43.7)$ \\
\hline CT & 38 (26.8) & $73(34.0)$ \\
\hline TT & $26(18.3)$ & $48(22.3)$ \\
\hline $\mathrm{C} / \mathrm{T}$ & $0.68 / 0.32$ & $0.61 / 0.37$ \\
\hline
\end{tabular}

$\mathrm{C} / \mathrm{T}$ indicates allele frequencies

Table 2: Clinical and metabolic characteristics of diabetic patients according to methylenetetrahydrofolate reductase C677T genotype. Data shown are the mean \pm SD

\begin{tabular}{lccc}
\hline Characteristics & $\begin{array}{c}\text { CC } \\
(\mathrm{n}=94)\end{array}$ & $\begin{array}{c}\text { CT } \\
(\mathrm{n}=73)\end{array}$ & $\begin{array}{c}\text { TT } \\
(\mathrm{n}=48)\end{array}$ \\
\hline Sex (female/male) & $38 / 69$ & $28 / 52$ & $17 / 32$ \\
Age (years) & $60.2 \pm 7.3$ & $58.7 \pm 8.4$ & $59.8 \pm 9.2$ \\
BMl (kg/m $\left.{ }^{2}\right)$ & $22.8 \pm 3.6$ & $24.3 \pm 4.1$ & $23.57 \pm 3.8$ \\
HbA1c (\%) & $8.4 \pm 3.1$ & $8.9 \pm 3.8$ & $8.1 \pm 2.7$ \\
Duration of diabetes (years) & $9.5 \pm 7.4$ & $10.2 \pm 8.2$ & $8.9 \pm 8.7$ \\
Family history of CAD (\%) & 14.6 & 13.8 & 14.1 \\
Current smoke ((\%) & 32.8 & 31.2 & 29.6 \\
Hypertension (\%) & 51.3 & 54.7 & 52.5 \\
Total cholesterol (mmol/l) & $5.48 \pm 0.94$ & $5.24 \pm 0.87$ & $5.32 \pm 0.97$ \\
HDL-cholesterol (mmol/l) & $1.45 \pm 0.62$ & $1.41 \pm 0.44$ & $1.37 \pm 0.53$ \\
LDL-cholesterol (mmol/l) & $3.14 \pm 0.92$ & $3.07 \pm 0.83$ & $3.20 \pm 0.90$ \\
Triglycerides (mmol/l) & $2.36 \pm 1.08$ & $2.49 \pm 0.96$ & $2.55 \pm 1.14$ \\
Serum creatinine (mg/dl) & $0.9 \pm 0.3$ & $0.9 \pm 0.5$ & $0.9 \pm 0.7$ \\
Overt proteinuria (\%) & 16.4 & 18.7 & 17.5 \\
Diabetic retinopathy (\%) & 23.7 & 22.6 & 24.8 \\
\hline
\end{tabular}

BMI- Body mass index; HDL- High-density lipoprotein;LDL- Low-density lipoprotein; CAD - coronary artery disease

Table 3: Association between methylenetetrahydrofolate reductase genotype and ischemic stroke in type $2 \mathrm{DM}$

\begin{tabular}{lcccc}
\hline Ischemic stroke & \multicolumn{4}{c}{ Genotype } \\
\cline { 2 - 5 } & CC & CT & TT & Total \\
\hline Positive & $45(47.9)$ & $36(49.3)$ & $38(79.2)$ & 119 \\
Negative & $49(52.1)$ & $37(50.7)$ & $10(20.8)$ & 96 \\
Total & 94 & 73 & 48 & 215 \\
\hline
\end{tabular}

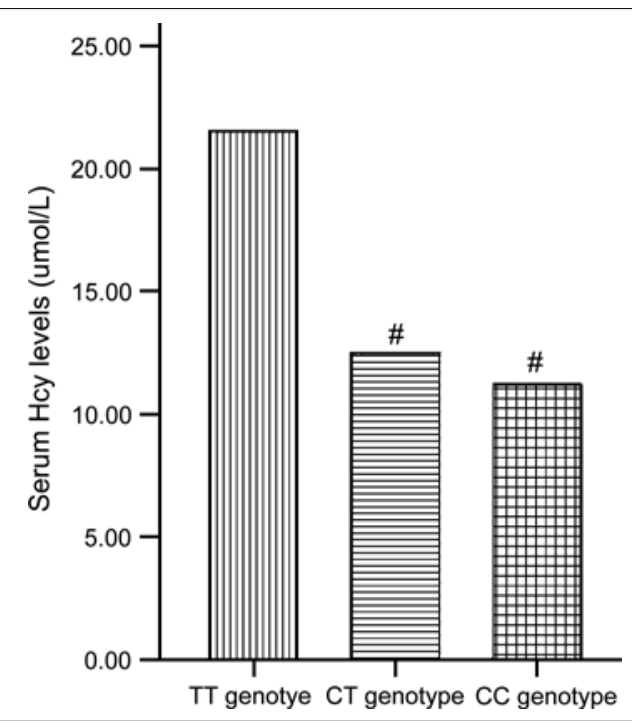

Figure 1: Serum Hcy levels according to methylenetetrahydrofolate reductase C677T genotype in diabetic patients. ${ }^{\# P} 0.01$ vs. TT genotype 
associated with a higher plasma Hcy levels in the diabetic patients. The difference in plasma Hcy levels between TT and CC, CT was statistically significant $(P<0.01)$, and there seemed to be a gradual change in serum Hcy levels according to the genotype.

Table 3 shows that the prevalence of ischemic stroke (79.2\%) were significantly higher in the diabetic patients with the TT genotype than those with the CT $(49.3 \%)$ or CC genotype $(47.9 \%)$. When we pooled the data for the CT and CC genotypes, the prevalence of ischemic stroke in the TT genotype was also significantly higher than in the CC + CT genotype $(\mathrm{OR}=4.04,95 \% \mathrm{CI}=1.95-8.34$, $P=0.0036)$.

\section{Logistic regression analysis}

Multiple linear regression analysis was used to examine the determinants of ischemic stroke. The TT genotype was significantly associated with the prevalence of ischemic stroke (OR $=2.84, P=0.002)$. The duration of diabetes mellitus, hypertension, smoking and hyperlipidemia were also found to be independent factors significantly associated with ischemic stroke [Table 4].

\section{Discussion}

The main finding in the present study was the evidence that both high plasma levels of Hcy and MTHFR C677T gene polymorphism are strongly associated with ischemic stroke in Chinese type 2 diabetic patients. In contrast, there were no significant difference in the frequency of the thermolabile form of MTHFR between diabetic patients and control subjects.

We observed a different genotype frequency in this study than the one reported in the previous studies. ${ }^{[7]}$ The population frequency of the C677T allele showed regional and ethnic variations. For example, In Europe, the mutant $\mathrm{T}$ allele frequency ranged from $24.5 \%$ in Germany to $43.8 \%$ in Italy. The prevalence of the mutant $\mathrm{T}$ allele is the lowest in Africa and the Middle East. ${ }^{[5]}$ In our study, the mutant $\mathrm{T}$ allele frequency was $32.0 \%$, and comparable to that in a Hong Kong (Chinese) population reported by Schneider et al..$^{[7]}$ These data suggest that this mutation has a relatively high frequency throughout the world and is highly prevalent in diverse ethnic groups and can be regarded as a balanced polymorphism that escaped natural selection. Such a polymorphism does not usually imply a serious lethal disorder. However, it can contribute to the pathogenesis of multifactorial diseases, such as diabetic angiopathy, in association with other factors.

In this study, the diabetic patients with TT genotype had higher plasma Hcy compared to those with the CC or CT genotype. This result is consistent with previous reports ${ }^{[8,9]}$ and showed the effect of the MTHFR gene C677T mutation on Hcy levels. Thus, the higher prevalence of ischemic
Table 4: Logistic regression analysis on multiple risk variables for ischemic stroke in type 2 DM

\begin{tabular}{lcc}
\hline & OR & $P$-value \\
\hline Male sex & 0.89 & 0.652 \\
Age(years) & 1.02 & 0.141 \\
BMI $\left(\mathrm{kg} / \mathrm{m}^{2}\right)$ & 1.10 & 0.01 \\
Smoking & 2.23 & 0.004 \\
Hypertension & 1.42 & 0.005 \\
Hyperlipidemia & 1.23 & 0.03 \\
Duration of & 1.12 & 0.005 \\
diabetes mellitus & & \\
MTHFRTT genotype & 2.84 & 0.001 \\
\hline
\end{tabular}

BMI- body mass index

stroke in the TT genotype can be attributed to the effect of MTHFR C677T genotype on Hcy levels. The results of logistic regression analysis in this study showed that the TT genotype was a significant risk factor for ischemic stroke in Chinese type 2 diabetes mellitus. The difference in Hcy levels among the MTHFR genotype seemed to imply that the Hcy levels account for the genotypic effect on ischemic stroke in the patients with diabetes mellitus.

The association of the homozygous C677T MTHFR genotype with atherosclerosis and thrombotic diseases has been the subject of many recent studies. A large body of data has showed that the genetic variant (C677T) in the MTHFR gene is associated with peripheral, cerebral and coronary arteries. ${ }^{[10-12]}$ However, not all researchers have found this association. ${ }^{[13-15]}$ Investigators have also failed to find any association between the genotype and obstructive coronary artery disease, ${ }^{[16]}$ atherosclerotic peripheral vascular disease, ${ }^{[17]}$ and ischemic atherosclerotic vascular disease. ${ }^{[18]}$

The different findings and conclusions obtained in the above studies may be partially explained by differences in study size and design. The other possible explanation is the methodological limitation of the current study. In addition, because these studies were conducted in different populations, there may be ethnic or environmental differences in terms of this relationship. However, if the risk conferred by the genotype is largely dependent on an additional factor such as serum folate, vitamins $B_{6}$ and $\mathrm{B}_{12}$ level, which may vary between populations, then the impact of the homozygous C677T MTHFR genotype might differ widely. Indeed, homocysteine levels are known to vary significantly between populations, ${ }^{[19]}$ and it is yet to be determined what proportion of this variation is attributable to diet and/or to genetic factors, including the C677T MTHFR genotype.

Epidemiological studies have demonstrated that hyperhomocysteinemia is an independent risk factor for stroke $e^{[20,21]}$ atherosclerosis in general, ${ }^{[22]}$ and carotid atherosclerosis in particular. ${ }^{[23]}$ The molecular mechanism by which Hcy promotes atherothrombosis is unknown. However, the underlying mechanism of how Hcy 
affects atherogenesis is unclear. The importance of hyperhomocysteinemia in predicting vascular complications in different locations has been highlighted by several studies ${ }^{[4,24]}$ Experimental evidence suggests that Hcy may contribute to both atherosclerotic and thrombotic processes by modulating vascular cell proliferation and promoting prothrombotic activity in the vascular wall. ${ }^{[25,26]}$ These effects of homocysteine may explain the close correlation between the C677T mutation of MTHFR which results in hyperhomocysteinemia and the presence of atherosclerotic disease in diabetes mellitus.

In conclusion, our present observations allow us to suggest that the C677T mutation of the MTHFR gene, which is known to cause mild hyperhomocysteinemia, is one of the genetic factors predisposing to development of ischemic stroke in type 2 diabetic patients. However, in order to create a system of preventive measures for vascular complications of type 2 diabetes mellitus, it is necessary to replicate the data in other ethnic populations. It will also be important to study prospectively that whether folate supplementation reduces the incidence of ischemic stroke in type 2 diabetes mellitus in individuals who carry the C677T allele.

\section{Acknowledgments}

This study was funded by Hubei Provincial Bureau of Health Science Foundation for Young Scholars (Grants QJX2008-29).

\section{References}

1. Fujishima M, Kiyohara Y, Kato I, Ohmura T, Iwamoto H, Nakayama K, et al. Diabetes and cardiovascular disease in a prospective population survey in Japan: The Hisayama Study. Diabetes 1996;45: S14-6.

2. Robbins AS, Manson JE, Lee IM, Satterfield S, Hennekens CH. Cigarette smoking and stroke in a cohort of US male physicians. Ann Intern Med 1994;120:458-62.

3. Stehouwer CD, Weijenberg MP, van den Berg M, Jakobs C, Feskens EJ, Kromhout D. Serum homocysteine and risk of coronary heart disease and cerebrovascular disease in elderly men: A 10-year follow-up. Arterioseler Thromb Vase Biol 1998;18:1895-901.

4. Frosst P, Blom HJ, Milos R, Goyette P, Sheppard CA, Matthews RG, et al. A candidate genetic risk factor for vascular disease: A common mutation in methylenetetrahydrofolate reductase. Nat Genet 1995;10:111-3.

5. Botto LD, Yang Q. 5,10-Methylenetetrahydrofolate Reductase Gene Variants and Congenital Anomalies: A HuGE Review. Am J Epidemiol 2000;151:862-77.

6. Whisnant JP, Basford JR, Bernstein EF, Cooper ES, Dyken ML, Easton JD, et al. National Institute of Neurological Disorders and Stroke Ad Hoc Committee. Classification of cerebrovascular diseases III. Stroke 1990;21:637-76.

7. Schneider JA, Rees DC, Liu YT, Clegg JB. Worldwide distribution of a common methylenetetrahydrofolate reductase mutation. Am J Hum Genet 1998;62:1258-60.

8. Kluijtmans LA, van den Heuvel LP, Boers GH, Frosst P, Stevens EM, van Oost BA, et al. Moleuclar genetic analysis in mild hyperhomocysteinemia: A common mutation in the methylenetetrahydrofolate reductase gene is a genetic risk factor for cardiovascular disease. Am J Hum Genet 1996;58:35-41.

9. Gudnason V, Stansbie D, Scott J, Bowron A, Nicaud V, Humphries S. C677T polymorphism in methylenetetra-hydrofolate reductase: Its frequency and impact on plasma homocysteine concentration in different
European populations. Atherosclerosis 1998;136:347-54

10. Gallagher PM, Meleady R, Shields DC, Tan KS, McMaster D, Rozen R, et al. Homocysteine and risk of premature coronary heart disease: Evidence for a common gene mutation. Circulation 1996;94:2154-8.

11. Izumi M, Iwai N, Ohmichi N, Nakamura Y, Shimoike H, Kinoshita M. Molecular variant of 5,10-methylenetetrahydrofolate reductase is a risk factor of ischemic heart disease in the Japanese population. Atherosclerosis 1996;121:293-4.

12. Christensen B, Frosst P, Lussier-Cacan S, Selhub J, Goyette P, Rosenblatt DS, et al. Correlation of a common mutation in the patients with premature coronary artery disease. Arterioscler Thromb Vasc Biol 1997;17:569-73.

13. Wilcken DE, Wang XL, Sim AS, MeCredie RM. Distribution in healthy and coronary populations of the methylenetetrahydrofolate reductase (MTHFR) C677T mutation. Arterioscler Thromb Vasc Biol 1996;16:878-82.

14. Schmitz C, Lindpaintner K, Verhoef P, Gaziano JM, Buring J. Genetic polymorphism of methylenetetrahyrofolate reductase and myocardial infarction: A case-control study. Circulation 1996;94:1812-4.

15. Verhoef P, Kok FJ, Kluijtmans LA, Blom HJ, Refsum H, Ueland PM, et al. The $677 \mathrm{C}-\mathrm{T}$ mutation in the methylenetetrahydrofolate reductase gene: Associations with plasma total homocysteine levels and risk of coronary atherosclerotic disease. Atherosclerosis 1997;132:105-13.

16. van Bockxmeer FM, Mamotte CD, Vasikaran SD, Taylor RR. Methylenetetrahydrofolate reductase gene and coronary artery disease. Circulation1997;95:21-3.

17. Deloughery TG, Evans A, Sadeghi A, McWilliams J, Henner WD, Taylor LM Jr, et al. Common mutation in methylenetetrahydrofolate reductase: Correlation with homocysteine metabolism and late-onset vascular disease. Circulation 1996;94:3074-8.

18. Markus HS, Ali N, Swaminathan R, Sankaralingam A, Molloy J, Powell J. A common polymorphism in the methylenetetrahydrofolate reductase gene, homocysteine, and ischemic cerebrovascular disease. Stroke 1997;28:1739-43.

19. Ganji V, Kafai MR. Third National Health and Nutrition Examination Survey. Demographic, health, lifestyle, and blood vitamin determinants of serum total homocysteine concentrations in the third National Health and Nutrition Examination Survey, 1988-1994. Am J Clin Nutr 2003;77:826-33.

20. Eikelboom JW, Hankey GJ, Anand SS, Lofthouse E, Staples N, Baker RI. Association between high homocyst(e)ine and ischemic stroke due to large and small artery disease but not other etiologic subtypes of ischemic stroke. Stroke 2000;31:1069-75.

21. Bostom AG, Rosenberg IH, Silbershatz H, Jacques PF, Selhub J, D'Agostino RB, et al. Nonfasting plasma total homocysteine levels and stroke incidence in elderly persons: The Framingham study. Ann Intern Med 1999;131:352-5.

22. Welch GN, Loscalzo J. Homocysteine and atherothrombosis. N Engl J Med 1998;338:1042-50.

23. Selhub J, Jacques PF, Bostom AG, D'Agostino RB, Wilson PW, Belanger AJ, et al. Association between plasma homocysteine concentrations and extracranial carotid-artery stenosis. N Engl J Med 1995;332:286-91.

24. Arruda VR, von Zuben PM, Chiaparini LC, Annichino-Bizzacchi JM, Costa FF. The mutation Ala677--> Val in the methylene tetrahydrofolate reductase gene: A risk factor for arterial disease and venous thrombosis. Thromb Haemost 1997;77:818-21.

25. Outinen PA, Sood SK, Liaw PC, Sarge KD, Maeda N, Hirsh J, et al. Characterization of the stress-inducing effects of homocysteine. Biochem J 1998;332:213-21.

26. Upchurch GR Jr, Welch GN, Fabian AJ, Freedman JE, Johnson JL, Keaney JF Jr, et al. Homocyst (e)ine decreases bioavailable nitric oxide by a mechanism involving glutathione peroxidase. J Biol Chem 1997;272:17012-7.

Accepted on 02-07-2009

Source of Support: Hubei Provincial Bureau of Health Science Foundation for Young Scholars (Grants QJX2008-29) , Conflict of Interest: None declared. 